\title{
The Design and Implementation of Novel Framework of the Internet of Things(IoT)
}

\author{
Yong $\mathrm{Li}^{1, \mathrm{a}}$ \\ ${ }^{1}$ Hengshui University, Hebei, China, 053000 \\ ylrs6688@sina.com
}

Keywords: Internet of Things(IoT); Novel Framework; Network Techniques; GIS System;

\begin{abstract}
With the rapid development and progress in computer science and information science technology, the combination of computer and real-world is needed. New technologies also provide novel techniques for environmental management. This paper establishes a framework for an Environmental Internet of Things (EIoT) and describes key technologies, including Wireless Sensor Network (WSN), network techniques, Geographic Information System (GIS), WebGIS, and distributed database techniques. Our proposed framework is consist of the following parts: (1)Sensors for touching, (2)network monitoring system, (3)database and data pool, (4)environmental information platform and management system(IPMS). The EIoT can also realize online environmental simulation and management and is currently being extended to the city, regional, and national levels. The design and implementation of EIoT will help us touch the real urban environment and assist to state-of-the-art technological solutions for the severe environment problems.
\end{abstract}

\section{Introduction}

While the world entered the urban society age in 2008, China's rate of urbanization reached $50 \%$ in 2012, nearly three times higher than the level in 1978 when the policy of economic reform and opening-up began. Rapid industrialization and urbanization require the initiation and promotion of the science or the study of urban environment, to find a way to integrate or couple urban development and eco-environment processes for sustainable cities. The development of the Environmental Internet of Things (EIoT) will be beneficial to the development of the science or the study of urban environment and to environmental management, the meaning is long and crucial. Higher urbanization rates have led to closer links between cities. Population growth and economic development have shown a notable trend to regionalization. Urban environmental problems are also becoming regional in nature. The arrival of the networked information era has resulted in the emergence of the 'digital city' (a connected community within the city that combines Information and Communication Technologies infrastructure). Urban environmental problems have also presented new features and changing trends, but their interrelated nature exacerbates the difficulty of environmental analysis, management, and protection. Conventional technology for environmental monitoring, simulation, and management seems to be isolated and inadequate at both temporal and spatial scales. The adoption of advanced technology and digital and networking methods in the study of urban environmental problems would be a major step forward in cognizing, analyzing, and solving increasingly serious urban environmental problems.

Internet of Things' applications are very extensive and include fields such as the smart grid, smart transportation, smart logistics, and e-health. At present, there are only preliminary applications in the environmental field, such as the online monitoring of pollutant sources and indoor environmental monitoring systems. However, the proposed Environmental Internet of Things is dealing with the environmental sciences and has many potential applications in environmental monitoring, simulation, and management. 


\section{The Architecture of Traditional IoT}

General Concepts of IoT. In a general observation, the traditional IoT could be divided in three parts: (1)Full sensing, meaning that RFID, sensors, and two-dimensional code are used to detect information about things anywhere and at any time. (2)Reliable delivery, as information is transmitted through the integration of all kinds of telecommunication networks and the Internet. (3)Intelligent processing, during which computing technologies, such as cloud computing and fuzzy recognition, are used to analyze and process massive amounts of data and intelligently control the 'things'. As a conclusion, we generally regard the IoT to be composed of three layers: the basic sensing layer for gathering the data needed, the network layer for data transformation, and finally the processing layer.

Crucial and Core Techniques of IoT The IoT is a network information system which combines the distributed technologies of information collection, transmission, and processing. Its characteristics include low-cost, miniaturization, low-power, flexible networking, and suitability for moving targets. The key technologies of the IoT are listed below: (1)Wireless sensor network: A WSN is a self-organized multi-hop network formed by wireless communication and is composed of a mass of low-cost tiny sensor nodes. Its purpose is to sense, collect, and process information from the sensed objects in the area covered by the network and to send this information to the observer. The sensor node consists of sensor, processor, wireless communication, an energy supply modules. (2)High speed network technology: This technology is used to achieve information sharing, interoperability, and distributed storage and computation of mass data. (3)GIS: GIS is a key technology in the urban environment field, providing a full set of management tools for location-specific environmental data, which can help decision-makers form specific policies. GIS has been widely adopted in the field of environmental protection, including environmental monitoring and assessment, emergency measures for accidental contamination, and environmental messages sharing. (4) Distributed database technique: This technique provides technical support for mass data processing within the EIoT. One distributed database can effectively realize distributed query and data updating. The IoT is continually expanding along with the development of the Internet and communications network, and its multi-functionality provides important technical support for environmental monitoring, simulation, and management.

\section{Our Proposed Framework}

EIoT Data-stream. A huge potential for environmental management exists in the expanding IoT. Having so many devices connected to a network will make it easier to control many aspects of daily life that have an impact on the environment and make their use more efficient. EIoT data begin with a network of environmental sensors, real-time environmental monitoring, visualization of environmental information, modeling of environmental analysis, spatialization of environmental management regions, and gridding of urban environmental management regions (Figure 1).

EIoT Application. The spatial units of the EIoT framework include nodes, stations, sample plots, cities, and the entire nation, and environmental sensors that have strong sensing capability and real-time functions are adopted to acquire long-term environmental information with high temporal resolution. The monitoring of various environmental factors based on the sensors at multi-spatial scales is shown in Figure 1. Based on the accumulation, pre-processing, and preliminary analysis of environmental data, we built several environmental models and used networking and digital analysis and visual representation to achieve smart environmental management. Since the creation of an environmental model requires the support of large amounts of data, we constructed fundamental databases including a basic urban geographic information database and urban infrastructure database. Other datasets which were compiled and stored include land-use changes, water quality, soil environment, acoustic environment, solid wastes, socio-economic background, ethnic minority cultures, and so on. The environmental information platform is based on mainstream GIS and the 
related spatial database, and environmental problems are analyzed, simulated, and predicted based on a professional analysis model of various environmental elements and problems. The EIoT datastream is shown in Figure 1. The framework of the EIoT is based on network technology, mobile communication networks, and WSN. WSN is used to sense, collect, and process environmental information; mobile communication networks are used to deliver mass environmental data to the information platform within a city; and the analysis and simulation results are published on the Internet via WebGIS and are shared by other cities. The application of network technology in the EIoT is shown in Figure 2. GIS, map server, and environmental information server are used to carry out urban environmental analysis, simulation, and information sharing. An urban environment dynamic simulation system is used to link and upgrade the early warning system for pollution accidents and to forecast and simulate the consequences.

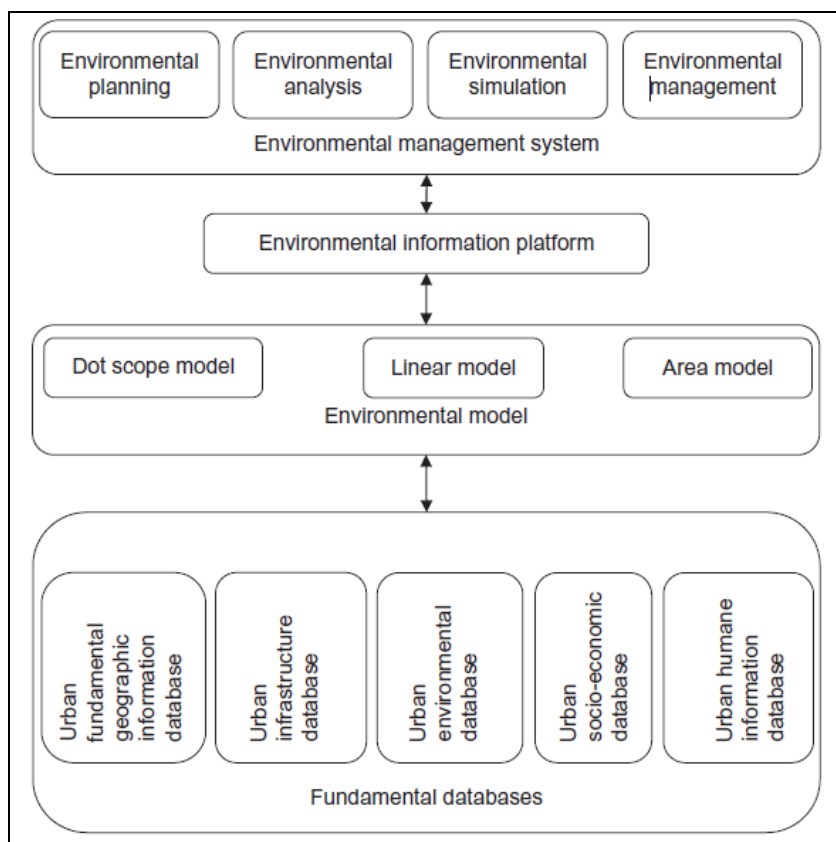

Figure.1 The Data-Stream

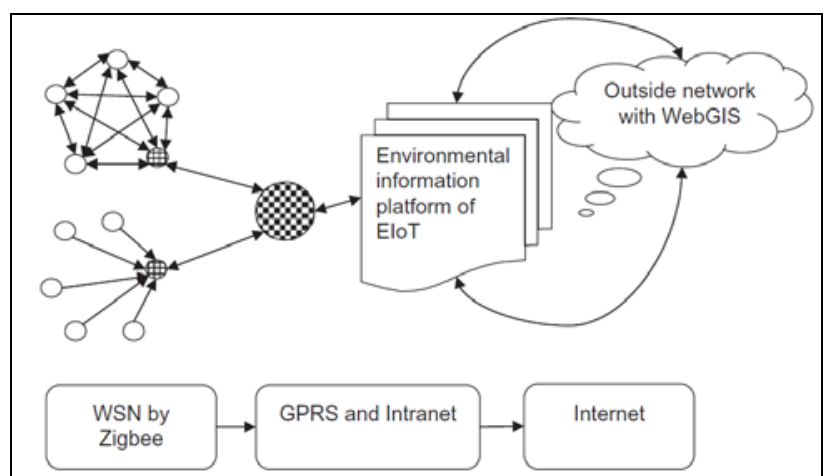

Figure.2 The Application of Network

\section{Construction of the Environmental IoT}

The Data Collection Procedure. Our EIoT uses wireless sensors to collect environmental data. The first step in EIoT construction was to design and make various wireless sensors for water, soil, atmosphere, noise, and wind monitoring. Each wireless sensor is fixed in one location and used to monitor the environment at, and around, that point; so in order to conduct real-time environmental monitoring over a large area, on the basis of our wireless sensors, we developed a wireless monitoring bicycle, car, boat, and plane, which provide flexible and convenient monitoring mode for water, soil, atmosphere, noise, and wind monitoring and make it possible to monitor the area where people cannot arrive easily, for example, a wireless monitoring boat can be used to monitor water quality in the center of a lake, and a wireless monitoring plane can be used to monitor atmosphere quality in the sky or survey environmental conditions of a forest.

The Data Transmission Procedure. The environmental data collected by sensors are transmitted to the central platform (the environmental information platform) via networks. In our EIoT, the transmission of environmental data from each node to the central platform at residential area level is via Zigbee. At the city level, the transmission of environmental data from monitoring station to the central platform is via GPRS, and at the regional and national levels, the transmission of environmental data from the central platform in a given city to the regional or national command central platform is via the Internet. 
The Data Reception and Simulation Procedure. Data reception and simulation is completed in the central platform. In our EIoT, the central platform is composed of several servers and monitoring equipment. Servers are used to store the environmental data from the sensors via networks and create various environmental models, and monitoring equipment is used to show in real time the monitoring and simulation results. In our EIoT, in the servers, we built databases for water quality, soil quality, atmosphere quality, noise environment, and wind environment as well as several other fundamental databases listed in the previous section. We constructed the EIoT at different spatial scales, including a typical residential area with high-density environmental sensor nodes, as well as other sample plots, and are gradually expanding it to monitor the whole sample city and eventually the whole of China. The multi-scale nature of our EIoT is shown in Figure 3.

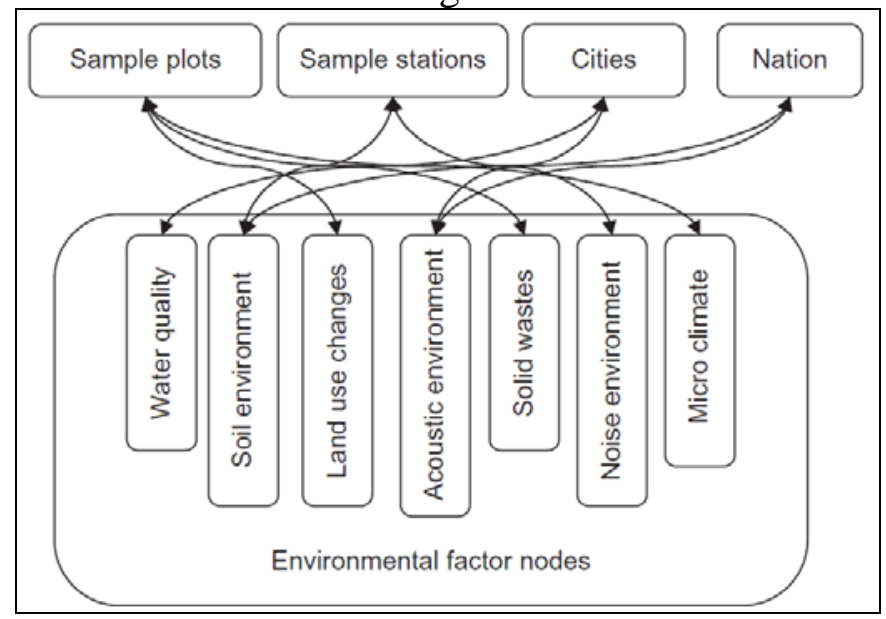

Figure.3 The Multi-Spatial Scales

\section{Summary and Conclusion}

Following integration of diverse information about the physical world, the EIoT can be used to simulate the evolution of the urban environment by building virtual cities, which can generate results which cannot be realized by experiment or engineering. This provides a new research platform for the study of urban environment and promotes the combination of micro and macro urban environmental research, the combination of research on a single urban environment with the regional geographical environment, and the combination of temporal and spatial scales. In conclusion, our EIoT covers not only all common environmental problems but it also has great flexibility and provides space to gradually expand the amounts and types of sensors and new technologies. A functioning EIoT at the residential-area level has been completed, and an EIoT at the city and regional levels is also being accomplished. Although the national-level EIoT presently only includes a few cities and sites, the command central platform at IUE will be used to gradually expand and monitor the whole of China..

\section{References}

[1] Amardeo C, Sarma JG. 2009. Identities in the future Internet of Things. Wireless Pers Commun. 49:353-363.

[2] Ergazakis E, Ergazakis K, Askounis D, Charalabidis Y. 2011. Digital cities: towards an integrated decision support methodology. Telemat Informat. 28:148-162.

[3] Yan B, Huang GW. 2008. Application of RFID and Internet of Things in monitoring and anti-counterfeiting for products. Proceedings of International Seminar on Business and Information Management, 2008 Dec 19; Wuhan, Hubei.

[4] Zhao J, Liu H, Dong R. 2008. Sustainable urban development:policy framework for sustainable consumption and production. Int J Sustain Dev World Ecol. 15:318-325. 